\title{
Research Paper: Occupational Therapists' Clinical Reasoning: A Qualitative Study
}

\author{
Narges Shafaroodi ${ }^{1}$, Mohammad Kamali ${ }^{2 *}$, Soroor Parvizi ${ }^{3}$, Afsoon Hassani Mehraban ${ }^{1}$
}

1. Department of Occupational Therapy, Faculty of Rehabilitation Sciences, Iran University of Medical Sciences, Tehran, Iran.

2. Department of Rehabilitation Management, Faculty of Rehabilitation Sciences, Iran University of Medical Sciences, Tehran, Iran.

3. Department of Pediatric Nursing, School of Nursing and Midwifery, Iran University of Medical Sciences, Tehran, Iran.

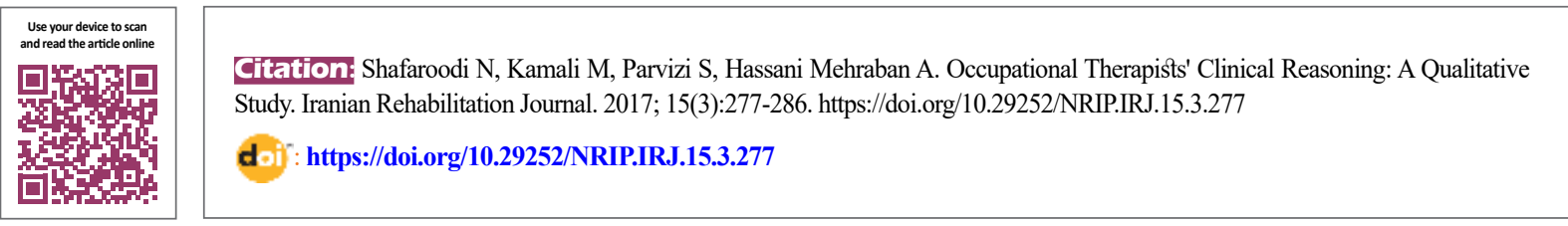

Article info:

Received: 16 May 2017

Accepted: 23 Jul. 2017

\section{Keywords:}

Context, Observable change, Social identity, Medical view, Holistic view, Professional identity, Grounded theory, Occupational therapist

\section{ABSTRACT}

Objectives: Clinical reasoning is at the heart of clinical work; it is affected by different field factors. A clear understanding of the reasoning process could solve practitioners' problems on how to make their underlying theories, assumptions and values more explicit. The aim of this research is to understand how clinical reasoning process is formed in the context of occupational therapists working in different clinical settings in Iran.

Methods: A purposeful and theoretical sampling of 15 occupational therapists working in clinical settings was performed through semi-structured interviews. All the data were recorded, transcribed, and analyzed using Strauss and Corbin's (2008) grounded theory approach and constant comparative analyses.

Results: Conceptual model was developed to explain the relationships among the main categories extracted through the grounded theory. Achieve an observable change emerged as the core category. Other important categories linked to the core category were, performing the continuum of clinical reasoning, context of clinical reasoning, and effective factors in clinical reasoning.

Discussion: Findings showed that achieving an observable change in the client was the main consequence of the action/interaction strategies. Some facilitating factors related to the therapist, helped to process reasoning with a holistic and client-centered view, while also helping to develop the self-belief and professional identity. The dominance of medical views and a lack of health insurance were two intervening factors that constrained the dynamics of clinical reasoning within the context of practice.

\section{* Corresponding Author:}




\section{Introduction}

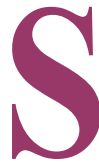

ince occupational therapy was established as a profession, it has evolved in response to the economic, political, medical and social demands of the time [1]. In the $21^{\text {st }}$ century, the aim of occupational therapists was to develop and perform purposeful clinical programs to maximize service users' independence and quality of life. Within such a context, the process of rehabilitation seeks solutions within unclear and changing situations $[2,3]$.

A considerable volume of research on the thinking processes and clinical reasoning of the occupational therapists has been conducted in developed countries over the last 40 years, and many researchers have attempted to explore the phenomenon and provide a clear definition of clinical reasoning [3-6]. Clinical reasoning lies at the heart of clinical practice and is a complicated, cognitive process for solving problems and making decisions $[5$, 7]. Moreover, clinical reasoning is a context-dependent process that guides occupational therapists to plan, direct, perform and reflect upon client care [8]. Clinical reasoning needs to be considered within authentic relationships, such as those held with clients and colleagues [9].

Clinical reasoning is dynamic and can be investigated in real-life situations and in response to current practice demands $[10,11]$. The different methods of reasoning explained in occupational therapy are as follows: interactive, conditional, narrative, procedural, pragmatic, ethical and generalization [11]. Some clinical/professional reasoning models have been stated to explain this complex phenomenon. Schell's Ecological Model (2006) defines professional reasoning as a process directly associated with therapy action, which is shaped by factors intrinsic to the therapist and client, as well as extrinsic factors in the practice context. Unsworth's (2004) conceptualized the three-tier hierarchy of clinical reasoning. At the top of this model is the therapist's moral beliefs and socio-cultural perspective (worldview) which influence and modify all the other modes of reasoning. Some researchers have employed these models and classifications and have studied clinical reasoning, manifesting the complications of clinical practice by identifying the various dimensions of occupational therapists' thought process $[5,6,12,13]$.

Previous and current research has proved that there are different factors affecting occupational therapists' practice. These factors include the balance or lack of it between personal and professional dynamics, politics related to the profession, historical background and existing expecta- tions in a specific clinical setting. Furthermore, it has been proven that therapists' reasoning is also affected by internal conditions such as therapists' own physical and mental experiences. These, in turn, are affected by external factors including the culture and practice contexts that constitute the worldview of the therapist $[4,12,14-19]$.

A more profound understanding of the factors affecting clinical reasoning helps in facilitating the design and implementation of culturally appropriate interventions that in turn can significantly lead to problem solving [20, 21]. This article aims to identify the clinical reasoning of occupational therapists within different clinical settings, considering the contextual factors affecting therapists' reasoning from a therapists' perspective.

\section{Methods}

\section{Research design}

This article constitutes a part of the thesis titled: The process of Clinical Reasoning in Occupational Therapists, which is guided by grounded theory. Grounded theory is used to recognize, explain and show relations between hitherto undiscovered variables with the aim of exploring a phenomenon from a new perspective, and thereby deriving conceptual and theoretical bases for the reasoning processes from the collected data. The decision of what and where to collect the next set of data is guided by the developing theory [22-25]. Clinical reasoning is a process formed by interactions of a contextual and interpersonal nature. Therefore, this qualitative research method can be used to explore the understanding and experiences of occupational therapists as part of the clinical reasoning process.

\section{Participants}

The research included participation by 15 occupational therapists who were selected using purposeful sampling method. Sampling started from an occupational therapy department in a school of rehabilitation and thereafter extended to three private rehabilitation centers, three university hospitals and day centers. Six practitioners and a faculty member with at least three years' experience were interviewed, after which theoretical sampling was conducted to get a clearer perception of the concept of the 'clinical reasoning setting' based on research data, categories and special properties appearing in each category. Subsequently, the effect of some factors, such as educational level and professional field on the clinical reasoning were studied. To do this, a complementary interview was conducted with eight other occupational 
Table 1. Participant demographic data

\begin{tabular}{|c|c|c|c|c|c|}
\hline No. & Gender & Area of Practice & Setting & Job Experience & Educational Level \\
\hline 1 & Female & Adult physical dysfunction & State clinic & 3 & MSc. \\
\hline 2 & Female & Adult physical dysfunction & State hospital & 12 & MSc. \\
\hline 3 & Female & Adult physical dysfunction & Private clinic & 22 & PhD candidate \\
\hline 4 & Male & Child physical dysfunction & Private clinic & 8 & BSc. \\
\hline 5 & Male & Child physical dysfunction & Private clinic & 5 & MSc. \\
\hline 6 & Male & Child physical dysfunction & State hospital & 7 & BSc. \\
\hline 7 & Female & $\begin{array}{l}\text { Child and adult physical } \\
\text { dysfunction }\end{array}$ & State hospital & 8 & MSc. \\
\hline 8 & Female & Adult physical dysfunction & State hospital & 19 & PhD candidate \\
\hline 9 & Female & Adult mental health & State clinc & 6 & MSc. \\
\hline 10 & Male & Child physical dysfunction & Private clinic & 10 & $\begin{array}{l}\text { PhD candidate in } \\
\text { neuroscience }\end{array}$ \\
\hline 11 & Male & $\begin{array}{l}\text { Child physical and mental } \\
\text { dysfunction }\end{array}$ & Private clinic & 10 & PhD candidate \\
\hline 12 & Male & Child physical dysfunction & State hospital & 13 & MSc. \\
\hline 13 & Male & $\begin{array}{l}\text { Child and adult physical } \\
\text { dysfunction }\end{array}$ & Private clinic & 16 & PhD candidate \\
\hline 14 & Female & Adult mental health & State hospital & 9 & MSc. \\
\hline 15 & Female & Child mental health & Private clinic & 5 & PhD candidate \\
\hline
\end{tabular}

therapists working at private outpatient settings and state hospitals (Table 1).

\section{Data gathering method}

After sampling had finished the study proceeded using the grounded theory approach [24]. Purposeful sampling was implemented to provide maximum diversity in participant selection. The interviews were conducted by a $\mathrm{PhD}$. candidate who had been working as a faculty member for 26 years. Some data (collated via telephone or in person) on the research and its aims were given to experienced faculty members of occupational therapy departments at three faculties of rehabilitation, as well as to occupational therapists working in hospital and outpatient settings. Eleven interviews took place at the rehabilitation faculties, and four interviews were further conducted at the interviewers' workplaces. Two of the interviews were interviewed twice in order to clarify some ambiguities.

Semi-structured interviewing was used as the main tool for data collection. Interviews started with general open-ended questions such as 'How do you begin with a client?'; 'Tell me more about the reasons for this decision?' and 'What factors influence your decisions? Subsequent questioning was based on the findings of the initial interviews and proceeded according to theoretical sampling using more precise and detailed questions. The interviews were for 20-70 minutes, and field notes were recorded by interviewer following each interview. After the sixth interview video permission was only issued for four sessions, and therefore, four therapy sessions were videotaped and observed.

The observer did not participate in any activity undertaken by the participant, and the video was utilized during interviews when the researcher asked interviews about their observed encounters. Feedback or prompting questions regarding observed behaviors served to prompt recollections and awareness of thinking and enable practitioners to verbalize their reasoning and explain the rationale for it. All interviews were taped and transcribed verbatim.

The data were analyzed using the constant comparative method. Interviews were conducted, recordings transcribed 
and data analyzed simultaneously. In fact, each interview provided direction for the ensuing one. All information extracted from the interviews, field notes and memos were analyzed using the Strauss and Corbin grounded-theory approach [24]. At this stage, the data were investigated line by line, after which the existing process was assigned, and the basic patterns were conceptualized.

Coding was performed using the participants' own words or by the reasoning of the research team according to concepts within the data. At the open coding level, the primary classification was formed and the codes were classified according to their properties. At the general level of analysis and interpretation, the researcher considered all conditions, actions or interactions and consequences, comparing different categories and creating subcategories that shared common properties within the same category. Exploring the relations between the categories and subcategories is the most important guide to the axial coding level of the theory. Selective coding was used to integrate categories into a theoretical scheme and to fill in any category that remained underdeveloped [26].

The base of the selected coding was the description of the main pattern that emerged within this study: 'reaching an observable change'. This is a repetitive variable that can create linkages among the categories. The data collection and analysis continued until data saturation occurred; that is, when no new category could be found through additional interviews. To maintain trustworthiness, several methods and maximum variation of sampling (choosing different participants from various settings, different academic degrees with various job experiences) were employed to enrich the obtained data. We also used member checking, peer checking, writing memo notes and reflexivity as some strategies to increase trustworthiness of the study results [25].

To increase data transferability, all research processes were documented and $30 \%-40 \%$ of the transcripts were peer reviewed by qualitative research specialists. Finally, three supervisors, a qualitative researcher and three participants discussed and revised the extracted categories and subcategories.

\section{Ethics}

The research proposal was approved by an ethics committee of Iran University of Medical Sciences. Informed consent was taken by all the participants. In order to maintain the participants' privacy and confidentiality, only the interviewer and the interviewee were present when the interviews were being conducted. Furthermore, in order to maintain anonymity, all manuscripts and audio files were coded. All participants were informed that they could withdraw from the study at any time and that they could take their audio files and transcript with them.

\section{Results}

Four main categories and 9 subcategories were extracted from our data via the grounded approach, enabling us to develop the conceptual model illustrated in Figure 1. Reaching an observable change was identified as the core category which occurred in the following three main categories: performing the continuum of clinical reasoning, context of clinical reasoning, and effective factors in clinical reasoning.

\section{Causal condition}

Causal condition is conceptualized as a set of events and situations that influence the emergence or development of reaching an observable change, which in turn can influence the clinical reasoning of an occupational therapist. The clients' awareness and understanding of their disease, as well as their related problems can influence the clinical outcome.

'That how much the patients have accepted their illnesses and how much they are familiar with the situation, how much information they have about their illnesses... are they willing to help themselves, this issue is so important that can affect our clinical reasoning. '

Some participants that worked in the hospital during the study declared that the acceptance of the patient's awareness of his own illness was impacted by social beliefs and the label that the patient extrapolated via such social beliefs. This means that the therapist has to spend some time with the patient correcting this viewpoint:

'Some patients really do not consider [mental] hospitalization as a treatment, but it seems to them like a kind of punishment. This is a negative matter and overshadows our activity. The patient thinks that he is guilty and he must be in jail for some time... we have to try hard to clear his mind of the statements he has heard and go along with the course of treatment.'

In some instances, intervention is performed based upon the limitations of the client in accessing occupational therapy services. In such situations, interventions may be carried out by the client or their caregiver after relevant training by a therapist. 


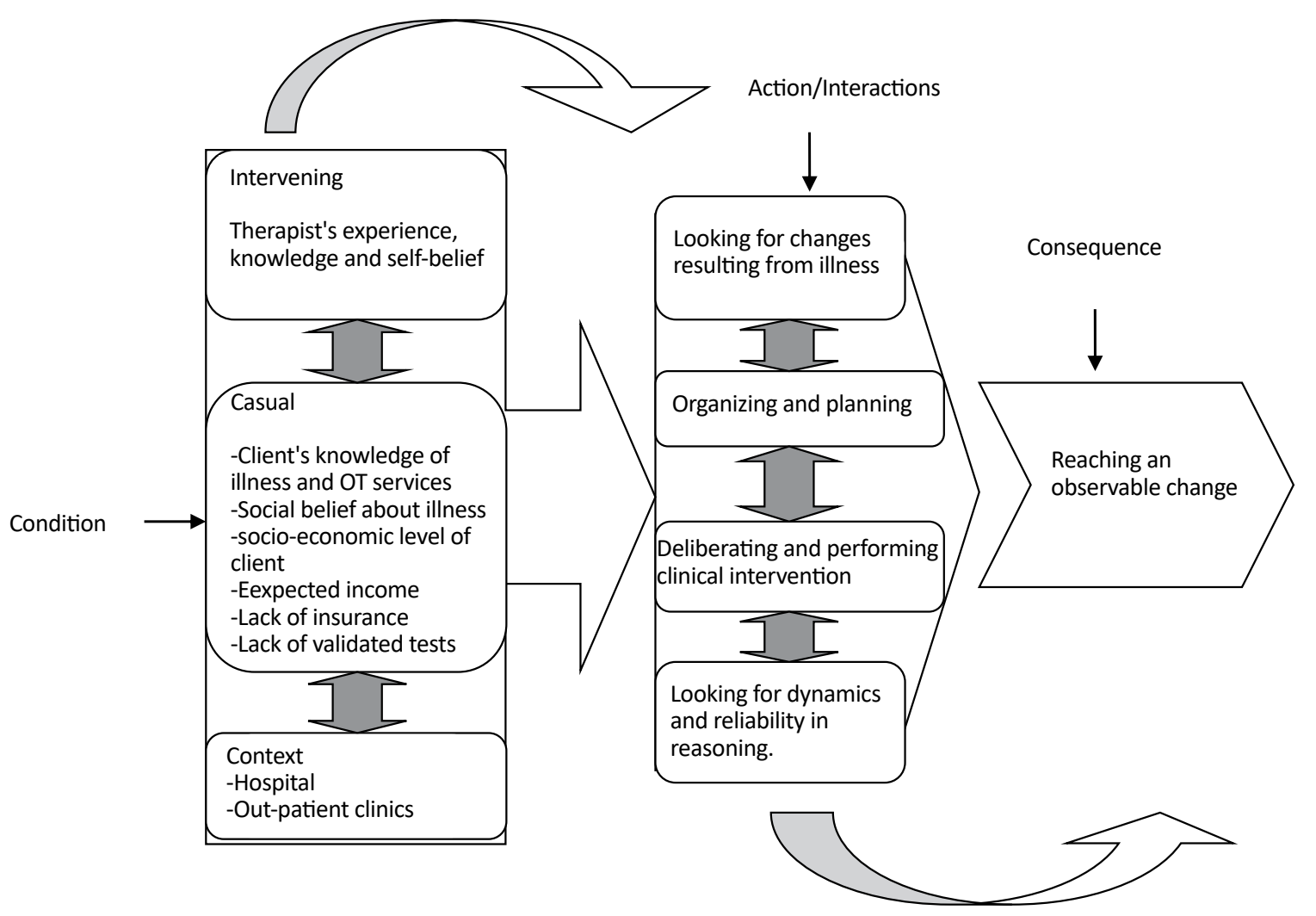

Figure 1. Conceptual model of clinical reasoning process of occupational therapy

Iranlan Rehabilicton Journal

'... We have training consultations, for instance for the clients who come from far away, ... He comes to the clinic in the morning and he does not have a place to stay for the night and he has to go back ... Assessment would be done, then I teach him or his caregiver how to perform the interventions and then answer their questions.'

The socio-economic level of the client has a main role in planning and determining the therapist's treatment goals. Most occupational therapists in this research determined their goals and started clinical intervention on the basis of their clients' economic situation and their ability to access the rehabilitation center. The financial status of the clients was able to determine the number of sessions held, the use of assistive devices and interventions.

'I think the financial status of the family affects the treatment. If she can attend regularly... she can have a good prognosis, but unfortunately some cannot continue because of their financial status ... then refer her to other centers or a government center.'

Extending our findings further, the conditions of the clinical environment, such as achieving expected income and lack of recognition of Occupational Therapy were effective in a goal setting and intervention.
'What affects the intervention is that insurance department [health cover] does not recognize our treatment. this old necessary field is not covered by insurance. The issue of self-funding and independent management of hospitals is a consideration. The rehabilitation funds cannot be compared with the surgical funds... we need to change our clinical program [in order to get more funds]. The quality of our work is also affected.'

All participants including those in the hospital and day center consider precise evaluation tools, such as standardized and validated tests necessary in order to remain critical and objective in their work and to observe targeted changes in the clients.

'One thing that I think may strengthen, or conversely weaken; our clinical reasoning process is that we cannot have very exact evaluation tools for client's problems... in order to have better reasoning.'

\section{Context}

Based on the conceptual model, context is a set of conditions in which a phenomenon is happening and people react through the action-interaction strategy. Occupational therapists begin their reasoning using a number of different strategies, which are affected by the conditions 
of the therapist and client within a specific context. For example, the context of the clinical environment determines the type of change. In a hospital context, the therapist considers the doctor's goals and focuses on resolving the symptoms, after which the, client will be discharged according to the doctor's decision. In the day center, the occupational therapist often tries to change the client's performance and environment. The process of clinical reasoning may continue for the changes considered by the occupational therapist, but the therapist is trying to challenge and create changes that are tangible for the client or the clinical team. Occupational therapists who were working in a hospital assessed the symptoms and effects in order to confirm the diagnosis provided by a doctor and any para-clinical information.

'The first matter in our job is the diagnosis by the doctor. It directs the occupational therapist's mind to a specific group... the things that are important after the doctor's prescription are the results of $x$-rays, MRI and then the background we obtain from the patient. These form a frame in our mind... and a judgment would form within us.'

Conversely, some experienced participants who were working in a day center did not consider the doctor's diagnosis first; they often established the effect of the disease on the client and his environment by applying different assessment methods in occupational therapy.

'... Assessment is important to us anyway, but the problem as to why the client has come is the most important. The diagnosis of the doctor may not be correct in some cases.'

\section{Intervening conditions}

Intervening conditions either facilitate or constrain action-interaction strategies in the specific context. Some participants declared that self-belief had a positive effect on the therapist's clinical reasoning from the very beginning.

'Most colleagues do not have a strong belief in the effectiveness of occupational therapy and rehabilitation. It is very important that the therapist should believe that he or she has the ability to bring about an effective cure to the client's condition.'

Intervening conditions such as experience and the educational level of the therapist were factors that affected clinical reasoning within the day centers. Those participants who were mentors or $\mathrm{PhD}$ students and who worked in day centers believed that they need to view their clients with a holistic perspective. This holistic ap- proach is effective in increasing client satisfaction within and throughout the treatment process.

'Depending on therapist's perspective... treatment can be continued because our patient(s) not only suffer from physical problems, but also suffer some emotional, mental and social problems; therefore, treatment program(s) can continue to resolve these problems.'

Some participants who were not faculty members believed that therapists should be in touch with new data in order to review their knowledge in practice. However, there are some limitations that allow easy access to new data and also they don't know how to use evidence based practice.

'Sometimes I am faced with a dead end... I know I am imperfect in my knowledge... I should not only maneuver on my own experiences, here it is important to work with new evidence .... to study or attend re-education classes can be effective but for all isn't possible.'

\section{Action interaction strategies}

Action interaction strategies are purposeful or deliberate acts which shape a phenomenon in some way. Looking for changes resulting from illness is the first step that the therapist uses to assess and gather data. The occupational therapist uses different assessment methods such as observation, interview, and implementation of clinical tests to diagnose the problems caused by illness. If the client has been referred by a medical physician, the occupational therapist would assess the effects of the disease on the client's activity, considering the doctor's diagnosis and paraclinical information.

'The first matter in our job is the diagnosis by the doctor. It directs the occupational therapist's mind to a specific group ... the things that are important after the doctor's prescription are the results of $x$-rays, MRI ... and then the background we obtain from the patient. These form a frame in our mind... and a judgment would form within us.'

Participants who work in day center stated that they provided information about the illness and occupational therapy services as an action-interaction strategy in order to obtain the trust and cooperation of the client and their family as an outcome.

'First we inform him ... explain to him what his problem is. You may not believe this, but most of the clients with stroke do not know that they have had a cerebral stroke. We have to explain ... We make the patient trust us.' 
Organizing and planning referred to those strategies that the participants used for organizing and determining their goals, intuitively and intelligently, when considering assessment results. The participants who work in hospital settings organize information based on their knowledge or intuitive experience irrespective of the clients' preferences.

'... A general view would be created in our mind by assessing the client's problem and which group [disorder] it fits into, in other words which group it goes in.'

The therapists who work at day centers intelligently organize the information according to the clients' preferences.

'We have to consider what the main problem is, we first consider the problem that the family has and then we try to assess [it]. We organize our goal and planning [with the family] considering the problem and try to solve the problem.'

Deliberation and intervention performance is another strategy that participants considered that were based on their world view, client's conditions and clinical setting.

'I directly perform my technical interventions during assessment of the client...often assessment and performing interventions are being done at the same time.'

Participants who were faculty members considered it necessary to do a holistic assessment before making any decision or performing interventions for the client.

'I assess the client in some treatment sessions ... I determine the treatment goals with client ... And then perform the interventions based on client's conditions.'

Looking for the dynamics and reliability of reasoning is the other strategy that participants start monitoring their decisions. Some participants start to evaluate their interventions through client feedback.

'First I evaluate and then I take a feedback ... Has the client had any progress that he/she expected from the treatment? Does he/she feel better?'

Participants who were either trainers or faculty members believed in evaluating their decisions continuously and reflecting upon both existing and new data in order to get good or ideal results.

'When we see good results, after a month or two, we evaluate again, and I check elementary results by that to see how much improvement we have had, what we need, what we did not pay attention to... in the case of no prog- ress, I usually try to think again to see if I can make some changes and I study new things too.'

Finally, through this dynamic and cooperative process, an observable change is made for the client.

\section{Discussion}

The study used grounded theory to explore the process of clinical reasoning among occupational therapists in Iranian context. According to the findings, some facilitating or constraining conditions influenced the therapist's reasoning strategies and achieved an observable change in the client. Some facilitating conditions related to the therapists, such as experience and acquisition of up to date knowledge and reflective skills, helped them to process their reasoning with a holistic and client-centered view. This issue influences the therapist's belief in the effectiveness of their role and their professional identity. However, the dominance of the medical perspective and lack of health insurance are intervening factors that constrained action/interaction strategies, as well as the dynamics of clinical reasoning in the practice context.

Dominance of the medical point of view in hospital setting led to the therapist merely finding and diagnosing the symptoms of the illness as an outcome seeking out changes resulting from an illness strategy. In day centers, therapists used this strategy to find the influence of the symptoms on functional performance; this issue might influence the therapist's belief in the effectiveness of their roles [27-29]. The client's awareness of the illness and occupational therapy services are either facilitated or constrained by therapist's action; also the acceptance of the problem and the winning the client's trust were those consequences that therapist expects to achieve for the client within the first phase of clinical reasoning.

In day centers, the therapist took into consideration the clients' awareness of the disease and their priorities and subsequently tried to establish and sustain a cooperative relationship with the client and family by making them aware of the illness and the services occupational therapy could offer them. The ability to communicate these requirements involved learning and utilizing a range of interpersonal skills as described by Taylor [30] in the intentional relationship model, as well as the findings of some other researchers $[31,32]$.

The participants declared that some clients and their families were affected by traditional societal beliefs about the illness due to cultural perspectives. The traditional view of the Iranian society regarding people 
with disability is piteous [33]. The implication that the clients have for their disease directly affects the process of therapy [34]. Occupational therapists who have been working at day centers categorize their evaluation data in consideration of their clients' conditions and priorities.

This issue might increase the level of cooperation between the therapist and client throughout the therapy process. The participants declared that it is not possible to work in such a way in the hospital setting and that occupational therapists determine goals in hospitals in cooperation with the treatment team. The results of this research confirm the findings of other research studies in showing that occupational therapists categorize and formulize the data from assessments under the effects of organizational constraints and that these have a significant impact on what is possible [32, 34, 35].

The participants declared that they deliberated and performed interventions to make changes according to the needs of the client or the expectation of the treatment team. The client's socio-economic circumstances and health insurance were conditions that determined the performance of clinical intervention. In this research, participants stated that they often referred their clients with low income to a government agency. A lack of health insurance limited the range of clinical options available to occupation therapists. Subsequently, many studies have pointed out the significance of this factor as part of the clinical reasoning process [36-39].

All the participants within the study were looking for dynamics and reliability while performing clinical interventions at a specific time in order to be sure of accuracy and the effect of performed intervention through consistent evaluation, feedback and reflection. Those participants with more clinical experience performed consistent evaluations according to a specific timetable and performed clinical interventions, gaining feedback regarding the clients and their families' satisfaction, as well as their treatment teams' expectations in day centers and hospitals. Those participants who were faculty members and trainers believed that clinical reasoning meant consistently analyzing, criticizing and exploring the data received from both previous and present conditions, while also reviewing the experiments and errors. When the therapist did not receive the expected result, it was necessary to review their errors and knowledge by searching for evidence that could potentially reduce the possibility repeating those errors.

The therapist used rethinking and critical thinking to give meaning to clinical reasoning. The clinical reason- ing process is dependent upon critical thinking and is influenced by a person's attitude, philosophical perspective and preconceptions [40-43]. The participants considered having feedback from the client, or the clients' families, and used valid tools for gathering data as important factors in decreasing errors in clinical reasoning. Receiving feedback and discussing the clinical activities can determine the level of success and failure [44, 45].

\section{Conclusion}

This research focused on achieving an observable change in the client using the action/interaction strategies. Some facilitating conditions related to the therapist helped to process reasoning with a holistic and clientcentered view, while also helping to develop the self-belief and professional identity. The dominance of medical views and a lack of health insurance were two intervening factors that constrained the dynamics of clinical reasoning within the context of practice.

In these circumstances, reviewing the curriculum of occupational therapy, updating knowledge by participating in re-educating course or workshops, and sharing experiences, understandings and negotiating outcomes in international, national or other health sciences conferences and congresses, might facilitate clinical reasoning process and develop a professional identity in this social context. The need for more qualitative and quantitative research on or related to this subject is raised if the greater knowledge that is to be obtained can make clinical services more effective in this context.

In this study, according to theoretical sampling, the data were gathered from different samples. The small numbers of participants in the samples limits the research and the results cannot be generalized to all occupational therapists.

\section{Acknowledgments}

This study was part of the third author's $\mathrm{PhD}$. dissertation in School of Nursing and Midwifery, Iran University of Medical Sciences. It is incumbent upon the authors to acknowledge the financial support offered by the Research Vice-Chancellor of Iran University of Medical Sciences. They also acknowledge the congenial contributions of the occupational therapists who participated in this study.

\section{Conflict of Interest}

The authors have no affiliations with or involvement in any organization with any financial interest or nonfinancial interest (such as personal or professional rela- 
tionships, affiliation, knowledge or beliefs) in the subject matter in this manuscript.

\section{References}

[1] Quiroga VAM. Occupational therapy: The first 30 years 1900 to 1930. Bethesda: American Occupational Therapy Association; 1995

[2] Mattingly C. What is clinical reasoning? American Journal of Occupational Therapy. 1991; 45(11):979-86. doi: 10.5014/ ajot.45.11.979

[3] Rogers J, Masagatani G. Clinical reasoning of occupational therapists during the initial assessment of physically disabled patients. The Occupational Therapy Journal of Research 1982; 2(4):195-219. doi: 10.1177/153944928200200401

[4] Mattingly C, Flemming MH. Clinical reasoning: Forms of inquiry in a therapeutic practice. Philadelphia: FA Davis; 1993.

[5] Rogers J. Clinical reasoning: The ethics, sciences, and art. American Journal of Occupational Therapy. 1983; 37(9):60116. doi: 10.5014/ajot.37.9.601

[6] Unsworth C. Using a head-mounted video camera to explore current conceptualizations of clinical reasoning in occupational therapy. American Journal of Occupational Therapy. 2005; 59(1):31-40. doi: 10.5014/ajot.59.1.31

[7] Audétat MC, Laurin S, Sanche G, Béïque C, Fon NC, Blais JG, et al. Clinical reasoning difficulties: A taxonomy for clinical teachers. Medical Teacher. 2012; 35(3):e984-e989. doi: 10.3109/0142159x.2012.733041.

[8] Boyt Scell BA. Professional reasoning in practice. In: Willard HS, Crepeau EB, Cohen ES, Schell BAB, editors. Willard \& Spackman's Occupational Therapy. Philadelphia: Lippincott Williams \& Wilkins; 2009.

[9] Loftus S. Rethinking clinical reasoning: time for a dialogical turn. Medical Education. 2012; 46(12):1174-8. doi: 10.1111/j.1365-2923.2012.04353.x

[10] Carrier A, Levasseur M, Bedard D, Desrosiers J. Clinical reasoning process: Cornerstone of effective occupational therapy practice. In: Söderback I, editor. International Handbook of Occupational Therapy Interventions. Berlin: Springer; 2015.

[11] Robertson L, Griffiths S. Clinical reasoning in occupational therapy: Controversies in practice. In: Robertson L, Griffiths S, editors. Problem Solving in Occupational Therapy. Hoboken, New Jersey: Wiley-Blackwell; 2012.

[12] Cohen S. Using cultural-historical activity theory to study clinical reasoning in context. Scandinavian Journal of Occupational Therapy. 2008; 15(2):82-94. doi: $10.1080 / 11038120701534975$

[13] Fleming $\mathrm{H}$. Aspects of clinical reasoning in occupational therapy. In: Willard HS, Crepeau EB, Cohen ES, Schell BAB, editors. Willard \& Spackman's Occupational Therapy. Philadelphia: Lippincott Williams \& Wilkins; 2009.
[14] Chapparo C. Clinical reasoning in occupational therapy. In: Higgs J, Jones MA, editors. Clinical Reasoning in the Health Professions. Amsterdam: Elsevier; 2008.

[15] Finlay L. Through the looking glass: Inter subjectivity and hermeneutic reflection. In: Finlay L, Gough B, editors. Reflexivity: A Practical Guide for Researchers in Health and Social Sciences. Hoboken, New Jersey: Blackwell Sciences; 2003.

[16] Ryan S, Higgs J. Teaching and learning clinical reasoning. In: Higgs J, Jones M, editors. In: Higgs J, Jones MA, editors. Clinical Reasoning in the Health Professions. Amsterdam: Elsevier; 2008.

[17] Schell B. Pragmatic reasoning. In: Schel B, Schell JW, editors. Clinical and Professional Reasoning in Occupational Therapy. Philadelphia: Lippincott\& Wilkins; 2008.

[18] Unsworth C. The clinical reasoning of novice and expert occupational therapists. Scandinavian Journal of Occupational Therapy. 2001; 8(4):163-73. doi: 10.1080/110381201317166522

[19] Whiteford G, Clair VWS. Occupation and practice in context. London: Churchill Livingstone; 2004.

[20] Schell B, Unsworth C, Schell J. Theory and practice: New directions for research in professional reasoning. In: Schel B, Schell JW, editors. Clinical and Professional Reasoning in Occupational Therapy. Philadelphia: Lippincott\& Wilkins; 2008.

[21] Turpin M, Iwama M. Professional reasoning in context. In Turpin MJ, Iwama MK, editors. Using Occupational Therapy Models in Practice: A Fieldguide. London: Churchill Livingstone; 2011

[22] Browne J. Grounded theory analysis: Coming to data with questioning minds. In: Minichiello V, Sullivan K, Greenwood R, Axford R, editors. Research Methods for Nursing and Health Science. London: Pearson; 2004.

[23] Charmaz K. Constructing grounded theory: A practical guide through qualitative analysis. Thousand Oaks, California: SAGE; 2006.

[24] Corbin JM, Strauss AL. Basics of qualitative research: Techniques and procedures for developing grounded theory Thousand Oaks, California: SAGE; 2008

[25] Polit DF, Beck CT. Nursing research: Principles and methods. Philadelphia: Lippincott Williams and Wilkins; 2003.

[26] Hallberg L. The "core category". Developed categories were integrated to form the of grounded theory: Making constant comparisons. International Journal of Qualitative Studies on Health and Well-being. 2006; 1(3):141-48. doi: 10.3402/ qhw.v1i3.4927

[27] Copley JA, Turpin MJ, King TL. Information used by an expert pediatric occupational therapist when making clinical decisions. Canadian Journal of Occupational Therapy. 2010; 77(4):249-56. doi: 10.2182/cjot.2010.77.4.7

[28] Harries P, Harries C. Studying clinical reasoning, Part 1: Have we been taking the wrong 'Track'? British Journal of Occupational Therapy. 2001; 64(4):164-68. doi: $10.1177 / 030802260106400402$

[29] Robertson L. Clinical Reasoning,part 1: The Nature of problem solving, a literature review. British Journal of Occupational Therapy. 1996; 59(4):178-82. doi: 10.1177/030802269605900407 
[30] Taylor R, Lee S, Kielhofner G. Therapeutic use of self: A nationwide survey of practitioners' attitudes and experiences. American Journal of Occupational Therapy. 2009; 63(2):198207. doi: 10.5014/ajot.63.2.198

[31] Schell B, Harris D. Embodiment: Reasoning with the whole body. In: Schel B, Schell JW, editors. Clinical and Professional Reasoning in Occupational Therapy. Philadelphia: Lippincott\& Wilkins; 2008.

[32] Stineman M, Rist P, Burk J. Through the clinician's lens: $\mathrm{Ob}-$ jective and subjective views of disability. Qualitative Health Research. 2009; 19(1):17-29. doi: 10.1177/1049732308327853

[33] Salehpour Y, Adibsereshki N. [Disability and Iranian culture (Persian)]. Tehran: University of Social Welfare and Rehabilitation Sciences; 2001.

[34] Kelly G. Understanding occupational therapy: A hermeneutic approach. British Journal of Occupational Therapy. 1996; 59(5):237-42. doi: 10.1177/030802269605900515

[35] Shafaroodi N, Kamali M, Parvizy S, Mehraban AH, O'Toole G. Factors affecting clinical reasoning of occupational therapists: A qualitative study. Medical Journal of The Islamic Republic of Iran. 2014; 28:8. PMID: 25250253

[36] Carrier A, Levasseur M, Bedard D, Desrosiers G. Clinical reasoning process underlying choice of teaching strategies: A framework to improve occupational therapists' transfer skill interventions. Australian Occupational Therapy Journal. 2012; 59(5):355-66. doi: 10.1111/j.1440-1630.2012.01017.x

[37] Unsworth C. Team decision-making in rehabilitation. American Journal of Physical Medicine and Rehabilitation 1996; 75(6):483-86. doi: 10.1097/00002060-199611000-00019

[38] Van-Ryan M, Burke J. The effect of patient race and socioeconomic status on physician's perceptions of patients. Social Science and Medicine. 2000; 50(6):813-28. doi: 10.1016/s02779536(99)00338-x

[39] Wainwright SF, McGinnis PQ. Factors that influence the clinical decision-making of rehabilitation professionals in long-term care setting. Journal of Allied Health. 2009; 38(3):143-51. PMID: 19753425

[40] Bannigan K, Moores A. A model of professional thinking: Integrating reflective practice and evidence based practice. Canadian Journal of Occupational Therapy. 2009; 76(5):34252. doi: $10.1177 / 000841740907600505$

[41] Carrier A, Levasseur M, Freeman A, Mullins G, Quénec'hdu $\mathrm{S}$, Lalonde $\mathrm{L}$, et al. Influence of societal and practice reasoning: a scoping study protocol. BMJ Open. 2013; 3(4):e002887. doi: 10.1136/bmjopen-2013-002887

[42] Robertson L, Griffiths S. Problem solving in occupational therapy. In: Robertson L, editor. Clinical Reasoning in Occupational Therapy: Controversies in Practice. Hoboken, New Jersey: Wiley-Blackwell; 2012.

[43] Sinclair K. Model for the development of clinical reasoning in occupational therapy [MSc. thesis]. Hong Kong: The Hong Kong Polytechnic University; 2003.

[44] Hambert TK. The use of clinical reasoning skills by experienced occupational therapy assistants. Pennsylvania: Pennsylvania State University; 2004.
[45] McKay E, Ryan S. Clinical reasoning story telling: Examining a student's story on the fieldwork of placement. British Journal of Occupational Therapy. 1995; 58(6):234-8. doi: $10.1177 / 030802269505800602$ 